\title{
Impacto do perfil socioeconômico de gestantes e parceiros na avaliação da qualidade do pré-natal
}

\author{
Impact of the socioeconomic profile of pregnant women and partners on the number of \\ prenatal consultations
}
Impacto del perfil socioeconómico de gestantes y parejas en la evaluación de la calidad de la atención prenatal

Lorenna Oliveira Menezes ${ }^{1 *}$, Teresa Virgínia Neves Floriano¹, Izailza Matos Dantas Lopes.

\begin{abstract}
RESUMO
Objetivo: Avaliar o impacto do perfil socioeconômico de gestantes e dos parceiros no número de consultas do pré-natal de recém-nascidos de uma maternidade de ensino no município de Aracaju-Sergipe. Métodos: Trata-se de um estudo transversal, retrospectivo e analítico. Foram analisados prontuários de crianças que necessitaram de internação em Unidade de Terapia Intensiva Neonatal (UTIN) no período compreendido entre janeiro de 2018 e junho de 2020. Foram incluídas variáveis dos genitores. Resultados: Entre genitoras dos 261 pacientes, houve uma média da idade de 25,4 anos e $56,1 \%$ possuíam atividade trabalhista. Em relação à escolaridade $56 \%$ possuíam ensino médio, 35,6\% eram analfabetas e $8,4 \%$ possuíam ensino superior. Em relação ao número de consultas pré-natal, $74,9 \%$ tiveram 6 ou mais consultas, enquanto 2,7\% não tiveram nenhuma. Ademais, 30,4\% dos parceiros possuíam vínculo empregatício. Conclusão: A população avaliada obteve baixa escolaridade e porcentagem reduzida de vínculo empregatício, o que contribui para a dificuldade de adesão do pré-natal assim como acompanhamento necessário, podendo resultar em riscos materno-fetais. Evidencia-se necessidade do pré-natal completo, além da tentativa de reduzir os fatores socioeconômicos que impactam negativamente.
\end{abstract}

Palavras-chave: Cuidado pré-natal, Fatores socioeconômicos, Recém-nascido.

\section{ABSTRACT}

Objective: To evaluate the impact of the socioeconomic profile of pregnant women and their partners on the number of prenatal consultations for newborns at a teaching hospital in the municipality of Aracaju-Sergipe. Methods: This is a cross-sectional, retrospective and analytical study. Medical records of children who will need admission to the Neonatal Intensive Care Unit (NICU) in the period between January 2018 and June 2020 were compensated. Variables from parents were included. Results: Among mothers of the 261 patients, there was an average age of 25.4 years and $56.1 \%$ had work activity. Regarding education, $56 \%$ had secondary education, $35.6 \%$ were illiterate and $8.4 \%$ had higher education. Regarding the number of prenatal consultations, $74.9 \%$ had 6 or more consultations, while $2.7 \%$ had none. In addition, $30.4 \%$ of the partners were employed. Conclusion: The evaluated population had a low level of education and a reduced reduction in employment, which contributes to the difficulty of prenatal adherence as well as necessary monitoring, which may result in maternal-fetal risks. The need for full prenatal care is evident, in addition to the attempt to reduce the socioeconomic factors that have a negative impact.

Keywords: Prenatal care, Socioeconomic factors, Newborn.

\section{RESUMEN}

Objetivo: Evaluar el impacto del perfil socioeconómico de las gestantes y sus parejas sobre el número de consultas prenatales de recién nacidos en un hospital universitario del municipio de Aracaju-Sergipe. Métodos: Se trata de un estudio transversal, retrospectivo y analítico. Se compensaron los registros médicos de los niños que necesitarán ingreso a la Unidad de Cuidados Intensivos Neonatales (UCIN) en el período comprendido entre enero de 2018 y junio de 2020. Se incluyeron variables de padres. Resultado: Entre madres de los 261

1'Universidade Tiradentes (UNIT), Aracaju - SE. *E-mail: loremenezes@hotmail.com 
pacientes, hubo una edad promedio de 25,4 años y el 56,1\% tenía actividad laboral. En cuanto a la educación, $56 \%$ tenía educación secundaria, el 35,6\% era analfabeta y el 8,4\% tenía educación superior. En cuanto al número de consultas prenatales, el $74,9 \%$ tuvo 6 o más consultas, mientras que el $2,7 \%$ no tuvo ninguna. Además, el 30,4\% de los socios estaban empleados. Conclusión: La población evaluada tenía un bajo nivel de educación y una reducción reducida del empleo, lo que contribuye a la dificultad de la adherencia prenatal y al necesario seguimiento, que puede resultar en riesgos materno-fetales. Es evidente la necesidad de una atención prenatal integral, además del intento de reducir los factores socioeconómicos que inciden negativamente.

Palabras clave: Atención prenatal, Factores socioeconómicos, Recién nacido.

\section{INTRODUÇÃO}

Com o objetivo de fornecer uma melhor atenção à saúde das mulheres no período gravídico e puerperal, o pré-natal surge como um elemento fundamental para os cuidados tanto maternos quanto fetais (VIELLAS EF, et al., 2014). Sabe-se que o Ministério da Saúde (MS) recomenda o quantitativo mínimo de 6 consultas de pré- natal, sendo uma consulta no primeiro trimestre de gestação, duas consultas no segundo trimestre e três consultas no terceiro trimestre. Nesse contexto, foi instituído em junho de 2000, o Programa de Humanização no Pré-Natal e Nascimento (PHPN), que objetiva reduzir as altas taxas de morbimortalidade materna e perinatal, amplificar o acesso ao pré-natal, determinar critérios para qualificar as consultas e promover o vínculo entre a assistência e o parto (BRASIL, 2000).

A assistência pré-natal refere-se ao acompanhamento da mulher gestante visando proporcioná-la uma gravidez e parto mais seguro e saudável. O acompanhamento materno-fetal ajuda a diagnosticar possíveis fatores de risco e definir tempo gestacional, acompanhando sua evolução e assegurando o bem estar da mãe e do feto, e ainda, reduzir as intercorrências que possam surgir ao longo deste processo e que podem levar a morbimortalidade materno e fetal (BONIM SSS, et al., 2020).

A importância do pré-natal inclui, também, a importância da participação do pai. O pai deve ser orientado a participar do processo de gestação da companheira, participando das consultas de pré-natal e das atividades do cotidiano, como comprar roupas, organizar, planejar as atividades habituais. O homem exerce papel importante no pré-natal, mas não somente pelo apoio e acompanhamento, pois através de sua presença neste período torna-se possível estabelecer sua identidade como "figura de pai", devendo sua participação ser estimulada (IFRS, 2018). No Brasil, ainda existem diferenças regionais no que tange à assistência pré-natal, incluindo o acesso, a prática realizada e a atuação dos profissionais, que acabam refletindo na qualidade do pré-natal, influenciando na evolução da gestação e puerpério (SERRUYA SJ, et al., 2004).

As consultas de pré-natal também são importantes no contexto da saúde mental, pois sabe-se que a gravidez e puerpério trazem grandes mudanças na vida familiar que podem desenvolver transtornos mentais e sofrimento psicológico. Assim, a ausência de apoio familiar, conflito e insatisfação conjugal, não ter apoio de seu parceiro, falta de apoio social no puerpério, exposição à violência por parceiro íntimo no ciclo gravídico puerperal e conflito familiar são fatores de risco para a mulher gestante que pode vir a desenvolver doenças psicomentais (ARRAIS AR e ARAÚJO TCCF, 2017).

$O$ tipo de doença pode trazer desfechos negativos à mulher e ao feto, uma vez que, quando não há preocupação em tratar o problema, aumentam os riscos das grávidas em adotarem maus hábitos comoo de tabagismo, alcoolismo e até mesmo drogas. Como consequência para a nova rotina da mãe, poderá ocorrer redução do autocuidado, como diminuir a quantidade e a qualidade alimentar e não aderir ao acompanhamento pré-natal, causando desnutrição, morbimortalidade neonatal, prematuridade, restrição do crescimento intrauterino e mortalidade materna (COSTA SP, et al., 2018).

Outro ponto importante relação ao pré-natal e sua adesão são as dificuldades encontrados no processo de trabalho logístico e burocrático das unidades de saúde, principalmente o agendamento tardio das consultas, desenvolvimento ineficaz das consultas e infraestrutura inadequada da unidade (AGUIAR FAR, et al., 2018). A deficiência no atendimento nas Unidades Básicas de Saúde é uma das maiores queixas dos usuários. 
A ausência ou escassez de profissionais na maior parte do tempo prejudicam o funcionamento adequado dessas unidades em relação à marcação das consultas (SALDANHA BL, 2020). Por outro lado, além das dificuldades de execução e acesso, a assistência ao pré-natal é influenciada pelo perfil socioeconômico das gestantes, principalmente no que diz respeito ao seguimento e adesão às orientações profissionais. Um dos principais fatores conhecidos é a idade materna (KASSAR SB, et al., 2006).

A Organização Mundial da Saúde define a adolescência como um período de transição biopsicossocial que corresponde a segunda década da vida, ou seja, dos 10 aos 19 anos. Incluso nesse intervalo, 0 Estatuto da Criança e do Adolescente (ECA), considera a adolescência como operíodo entre 12 a 18 anos, e em alguns casos excepcionais quando disposto na lei, o estatuto é aplicável até os 21 anos (BRASIL, 1990). Puérperas adolescentes e adultas jovens, no serviço público, na maioria dos casos, estão no mesmo nível socioeconômico. No entanto, percebe-se a tendência a fatores desfavoráveis às adolescentes como menor renda familiar e per capita, número reduzido de consultas no pré-natal e baixa escolaridade. Nos últimos anos, a maioria das publicações envolvendo a temática da não realização do pré-natal, considerando todas as faixas etárias maternas, elege que a ausência se deve, principalmente, a fatores socioeconômicos como baixa renda e baixo nível de escolaridade, além das dificuldades de custo para transporte (ROSA CQ, et al., 2014).

Levando em consideração a grande relevância da temática abordada, este trabalho teve por objetivo avaliar o impacto do perfil socioeconômico no número de consultas pré-natal, assim como seus principais determinantes envolvidos, em uma maternidade de ensino no Estado de Sergipe.

\section{MÉTODOS}

Trata-se de um estudo transversal, retrospectivo e analitico realizado em uma maternidade de ensino no município de Aracaju - Sergipe. Foram analisados prontuários de crianças nascidas em um hospital que necessitaram de internação em Unidade de Terapia Intensiva Neonatal (UTIN), no período compreendido entre janeiro de 2018 a junho de 2020. Utilizou-se um instrumento para a pesquisa elaborado pelos pesquisadores, que possuía variáveis epidemiológicas maternas e dos respectivos parceiros.

A amostra utilizada para coleta de dados foram prontuários de papel dos récem-nascidos egressos da UTIN que estavam de acordo com os critérios de inclusão e faziam seguimento no ambulatório de especialidades da maternidade do estudo. Os prontuários de crianças que não tenham sido internadas na UTIN, bem como os que não correspondiam ao período delimitado e/ou sem variáveis analisadas, tornaramse critérios de exclusão.

As variáveis maternas corresponderam à Idade, Escolaridade, Atividade Remunerada/trabalhista e Número de consultas Pré-natais. Em relação à escolaridade houve uma subdivisão em: Analfabeta, Fundamental completo, Fundamental incompleto; Ensino médio Completo e Ensino Médio Incompleto; Ensino superior Completo e Ensino superior incompleto. Em relação à atividade trabalhista foi coletado se possuía ou não. Em relação ao número de consultas no pré-natal, houve uma subdivisão em relação ao número de consultas, como: nenhuma; de uma (1) a cinco (5); seis (6) ou mais. As variáveis utilizadas dos parceiros foram a Idade e a Atividade Trabalhista (se possuía ou não).

Os dados foram compilados no programa Microsoft Office Excel. Foram obtidas variáveis qualitativas e quantitativas. Para as variáveis qualitativas a análise descritiva procedeu com a categorização dos dados e obtenção das respectivas frequências e percentuais e o cálculo da média, mediana, desvio padrão, mínimo e máximo das variáveis quantitativas. Foi realizada uma análise inferencial com o cruzamento entre variáveis categóricas e/ou quantitativas. Para verificar a associação entre tais variáveis qualitativas categóricas foi utilizado o teste Qui- quadrado, quando houve frequência observada menor que 5 utilizou-se o Teste Exato de Fisher (FISHER RA,1922). Utilizou-se o Kruskal-Wallis para comparações múltiplas e o teste de Dunn para determinar quais desses grupos são diferentes, e foram calculadas as medianas para cada grupo (PEARSON K, 1920).

O tamanho da amostra foi calculado com base da média de atendimento mensal do ambulatório de especialidades da maternidade do estudo considerando um erro de 5\% e um nível de confiança de 95\%. 
Para a realização deste cálculo foi considerado uma população finita $(\mathrm{N})$ de 200 pacientes, desvio padrão de 0,3 (alfa), $Z$ (variável normal padronizada associada ao nível de confiança $=1,96$ ) e o erro amostral de 0,05 (E). Resultando em um total de 136 prontuários, que foi incrementado para 261 participantes para compensar qualquer perda potencial e aumentar o poder do estudo. O projeto foi submetido à apreciação e avaliação do Comitê de Ética em Pesquisa (CEP) da Universidade Tiradentes. Após aprovação pelo CEP em 10 de agosto de 2020, pelo parecer de número 4.203.918, a pesquisa foi realizada mantendo-se nos padrões exigidos pela instituição. Não foi necessário Termo de Consentimento Livre e Esclarecido (TCLE), pois não houve contato com o paciente, visto que se trata de um estudo retrospectivo.

\section{RESULTADOS}

No estudo foram admitidos 261 pacientes com média de idade de 25,4 anos, com a idade variando entre 13 a 43 anos. Mães adolescentes ocuparam 15,31\% do total de pacientes que possuiam esses dados nos prontuários. Destas, a média de idade das adolescentes foi de 16,53 anos, com uma variação de 13 a 18 anos. O número médio de consultas no pré-natal foi 6,8 - variando entre 0 e 13 consultas. Dentre as pacientes que fizeram o pré-natal (219), $74,9 \%$ realizaram seis o mais consultas, enquanto $22,4 \%$ realizaram entre 1 a 5 consultas. Por fim, apenas 2,7\% das pacientes não realizaram consulta pré-natal (Tabela 1).

Tabela 1 - Estatística descritiva dos dados epidemiológicos.

\begin{tabular}{lccccccc}
\hline Variável & $\mathbf{N}(\%)$ & Omissos(\%) & Média & Mediana & Desvio Padrão & Mínimo & Máximo \\
\hline Idade da mãe & $\begin{array}{c}235 \\
(90,0)\end{array}$ & $26(10,0)$ & 25,4 & 25,0 & 6,7 & 13,0 & 43,0 \\
$\begin{array}{l}\text { Número de } \\
\text { pré-natal }\end{array}$ & $\begin{array}{c}219 \\
(83,9)\end{array}$ & $42(16,1)$ & 6,8 & 7,0 & 2,7 & 0,0 & 13,0 \\
\hline
\end{tabular}

Fonte: Menezes LO, et al., 2020.

Dentre as variáveis, foram analisados os resultados relacionados à escolaridade materna. Dos prontuários analisados, $86,2 \%$ possuíam dados sobre a escolaridade materna. Dentre os prontuários com dados completos 56\% possuíam Ensino Médio (completo ou incompleto), 35,6\% constavam como Analfabetas ou Ensino Fundamental (incompleto ou completo) e, por fim, 8,4\% constavam como Ensino Superior (completo ou incompleto). A avaliação da execução ou não de atividade remunerada estava presente em 233 prontuários (Tabela 2). No grupo das pacientes que não possuíam atividade remunerada, cerca de $72,1 \%$ eram Donas de Casa, enquanto as demais tiveram o percentual dividido entre estudantes e desempregadas.

Tabela 2 - Estatística descritiva dos dados epidemiológicos, considerando o número total de prontuários analisados.

\begin{tabular}{lcc}
\hline Variável / Categorias & Frequência & Porcentagem (\%) \\
\hline Escolaridade materna & & \\
- Analfabeto ou Ensino Fundamental (incompleto ou completo) & 80 & 30,7 \\
- Ensino Médio (incompleto ou completo) & 126 & 48,3 \\
- Ensino Superior (incompleto ou completo) & 19 & 7,3 \\
- Ignorado & 36 & 13,8 \\
\hline Atividade Remunerada & & \\
- Sim & 79 & 30,3 \\
- Não & 154 & 59,0 \\
- Ignorado & 28 & 10,7 \\
\hline Pré-natal & & \\
- 0 & 6 & 2,3 \\
- a 5 & 49 & 18,8 \\
- ou mais & 164 & 62,8 \\
- Ignorado & 42 & 16,1 \\
\hline
\end{tabular}

Fonte: Menezes LO, et al., 2020. 
Em relação aos parceiros, dos prontuários analisados, $84,7 \%$ possuiam informações da idade dos mesmos, cuja variação foi entre 17 e 58 anos, com média de 29,66 anos. Ao analisar a faixa etária separadamente, observou-se a predominância do intervalo de 20 a 29 anos, ocupando 50,22\% (Tabela 3).

Tabela 3 - Estatística descritiva dos dados epidemiológicos do parceiro, considerando a faixa etária.

\begin{tabular}{lccc}
\hline Variável no prontuário & $\mathbf{N}(\%)$ & Faixa etária & $\mathbf{N}(\%)$ \\
\hline Sim & $221(84,7 \%)$ & até 19 anos & $16(7,2 \%)$ \\
& & 20 a 29 anos & $111(50,2 \%)$ \\
& & 30 a 39 anos & $66(29,9 \%)$ \\
Não & 40 a 49 anos & $24(10,9 \%)$ \\
\hline
\end{tabular}

Fonte: Menezes LO, et al., 2020.

Levando em consideração a atividade remunerada, 224 protuários possuiam essa variável, sendo destas $86,4 \%$ afirmando algum tipo de vínculo trabalhista (Tabela 4).

Tabela 4 - Estatística descritiva dos dados epidemiológicos do parceiro, considerando o vínculo trabalhista/remunerado.

\begin{tabular}{lc}
\hline Vínculo trabalhista/Atividade remunerada & $\mathbf{N}(\%)$ \\
\hline Sim & $194(86,6 \%)$ \\
Não & $30(13,4 \%)$ \\
Total & 224 \\
\hline
\end{tabular}

Fonte: Menezes LO, et al., 2020.

\section{DISCUSSÃO}

É indispensável a questão do pré-natal como item de proteção para a mãe e o bebê. A correlação dos cuidados pré- natais adequados com melhores resultados perinatais, tanto em puérperas quanto em recémnascidos, são de notória relevância. A associação de outros fatores paternos e maternos determinantes como condições socioeconômicas, idade materna, número de gestações, escolaridade e estado civiltambém podem impactar na promoção de saúde da mulher e, consequentemente, repercutir na sociedade (MARCHETTI JR, et al., 2020). Em relação à idade materna, a variação de 13 a 43 anos das pacientes inclusas na pesquisa revelou valores extremos de idade com influência em diversas esferas. Muitas vezes mulheres mais novas tardam ao procurar assistência médica e realizar o pré-natal de acordo com o preconizado pelo Ministério da Saúde (mínimo de seis consultas ao longo da gestação).

Uma pesquisa feita pela Universidade Estadual de Maringá (PR) demonstrou que a idade materna e o nível inferior de escolaridade estão associadas à maiores taxas de natimortalidade e assumem especial relevância por sua interrelação com os outros fatores associados ao óbito fetal. As consequências dessa ação podem ser das mais variáveis possíveis, desde baixo peso ao nascer, infecções perinatais ou, até mesmo, comprometimentos neurológicos irreversíveis para o bebê (GRAVENA AAF, et al., 2013).

A gestação na adolescência, na maioria das vezes, não é programada e termina por correlacionar-se diretamente com o grau de escolaridade materna. Grande parte das adolescentes gestantes não finalizaram os estudos e, por consequência das novas responsabilidades de uma maternidade, findam em não priorizálo mais, tornando-se um fator de afastamento e dificuldade (DIAS ACG, et al., 2010). Na realização desta pesquisa foram coletados dados em relação à faixa etária materna, na qual observou-se que $15,31 \%$ do total de pacientes, 261 mães, eram adolescentes. Dessas, a média de idades das mães adolescentes foi de 16,53 anos. Em uma pesquisa realizada pelo Departamento de Ciências Médicas da Universidade Estadual de Campinas, foram analisados os nascimentos de filhos de adolescentes ocorridos na cidade de Campinas em 2001. Dos resultados encontrados no total de 14.444 partos, as adolescentes eram $17,8 \%$ das mães avaliadas e 0,7\% eram menores de 15 anos (CARNIEL EF et al., 2006).

Nesse contexto comparativo, segundo o IBGE (Instituto Brasileiro de Geografia e Estatística) em 2000, o grupo de mulheres de 10 a 19 anos, definido pela Organização Mundial da Saúde (OMS) como período da 
adolescência, representou $10,3 \%$ da população brasileira, e a proporção de gravidez nessa faixa etária foi de $23,5 \%$, sendo $0,9 \%$ em menores de 15 anos e 22,6\% nas mulheres de 15 a 19 anos (IBGE, 2013).

Assim, é possível observar que os valores colhidos nesta pesquisa realizada na maternidade Santa Isabel, em Aracaju-SE, são semelhantes aos dados de outras localidades e aos dados do IBGE, com uma pequena variação. Preconizado pelo MS, o pré-natal deve ser feito com um mínimo de 6 consultas. Estas são subdivididas em: uma no primeiro semestre, duas no segundo semestre e três no terceiro trimestre. $O$ impacto da idade materna no número de pré-natais realizados é de grande relevância. O presente estudo comprovou que $74,9 \%$ das pacientes realizaram 6 ou mais consultas no pré-natal em contraste com 2,7\% que não realizaram nenhuma consulta, sendo esse um resultado satisfatório, mas ainda não-ideal.

Em relação à frequência do pré-natal, as adolescentes sempre estão em desvantagem nesse tipo de assistência quando comparadas a outros grupos etários, isso faz com que haja um retardo do início à assistência pré-natal, que pode levar a uma maior exposição dos seus conceptos a riscos evitáveis (FARIAS $\mathrm{RV}$, et al., 2020).

A correlação entre as variáveis do nível de escolaridade e perfil empregatício também foi elucidada nos prontuários analisados. Verificou-se que $30,7 \%$ das pacientes avaliadas eram analfabetas ou possuíam apenas Ensino Fundamental completo ou incompleto, o que teve significativa variação comparada a um estudo realizado em Maceió - Alagoas. Nesse estudo, que comparou condições socioeconômicas e reprodutivas entre mães adolescentes e adultas jovens, com grupo amostral de 500 pacientes, $81,2 \%$ das pacientes eram analfabetas ou possuíam menos de 4 anos de estudo. Essa análise é consistente visto que o nível de escolaridade é componente da avaliação socioeconômica e se correlaciona também com a renda familiar. Foi percebido, também, a ampla variação ocorreu entre estados pertencentes à uma mesma região. Tal análise serve para ampliar as discussões das significativas diferenças no perfil da gestante que participa do serviço público de assistência pré-natal. (KASSAR SB, et al., 2006).

Assim a condição socioeconômica torna-se um dos motivos que influencia diretamente a não procura por cuidados relacionados à promoção, proteção e prevenção à saúde, por não serem vistos como prioridades em maior parte do tempo (SALDANHA BL, 2020). A prevenção de complicações obstétricas e as ações educativas desenvolvidas no pré-natal são essenciais para o acompanhamento e orientação da mulher no ciclo grávido-puerperal, ressaltando-se o papel preponderante da equipe obstétrica neste processo (SPINDOLA T, et al., 2006).

Existem inúmeras formas de realizar um trabalho educativo, destacando-se as discussões em grupo, conhecidas popularmente como grupo de gestantes. Esta metodologia educativa pode ser bastante explorada, pois objetiva a promoção da saúde e consequentemente a prevenção de doenças, buscando a participação das mães na concretização do autocuidado (GUERREIRO M, et al. 2014).

Assim, segundo Cardoso RF, et al. (2019) as gestantes que participam de grupos sentem-se mais seguras sobre o processo gestacional e aos cuidados pós-parto consigo e com o bebê, sendo essa estratégia uma forma de vínculo com a unidade de saúde e de continuidade das consultas do pré-natal. A assistência pré-natal engloba também a presença dos parceiros das gestantes, cujos estudos revelam que as suas participações produzem reflexos positivos em várias esferas da assistência ao nascimento e parto (CARDOSO VEPS, et al., 2018).

Ao traçar um perfil epidemiológico dos parceiros submetidos a tal pesquisa, observou-se uma variação de idade entre 17 e 58 anos (média de 29,6 anos), sendo esse valor próximo à média da idade das mães, de 25,4 anos. A idade também vicula-se com outra variável relevante: o trabalho. A atividade remunerada dos acompanhantes torna-se um importante pilar nessas famílias, uma vez que a maior parte das mães submetidas à pesquisa não possuiam esse tipo de vínculo empregatício. Assim, analisou-se que $86,4 \%$ dos parceiros possuiam algum tipo de atividade remunerada com vínculo trabalhista. Ao avaliar a adesão das gestantes ao pré-natal, em outra pesquisa realizada na cidade de Pelotas, no Rio Grande do Sul, observouse que as menores escolaridades (quatro a sete anos e menos de quatro anos) estavam estatisticamente mais prevalentes no contexto de não realização do pré-natal. 
Ademais, a distribuição do perfil de emprego das pacientes também influencia, visto que $59 \%$ possuem atividades não remuneradas, sendo maioria nesse estudo. Dessa forma, conclui-se que a minoria das pacientes tem remuneração, podendo ser com vínculo formal ou informal, assim como a minoria possui Ensino Superior (completo ou incompleto), somando 7,3\%. Por conseguinte, infere-se que a escolaridade baixa e a ausência de atividade remunerada impactam negativamente na assistência pré-natal, variando de acordo com o município estudado.

\section{CONCLUSÃO}

A presente pesquisa propôs discutir o impacto do perfil socioeconômico de gestantes e parceiros na avaliação da qualidade do pré-natal. Através da avaliação de prontuários e análise estatística, foi possível avaliar variáveis diversas que interferem positiva e negativamente no contexto do pré-natal. Assim, é importante ressaltar que a baixa condição socioeconômica é um fator que interfere na má adesão às consultas do pré-natal. Os cuidados e as orientações no período pré-natal devem ser seguidos por todas as gestantes. É notória a necessidade de políticas públicas que ampliem e enfatizem a informação do acompanhamento de um profissional de saúde desde o início da gestação. Campanhas educacionais que englobem a importância da escolaridade para a formação do cidadão também devem ser influenciadas, principalmente no público mais jovem, a fim de que a gestação não se torne um obstáculo para essas gestantes. As principais dificuldades encontradas na presente pesquisa foram o preenchimento incorreto ou incompleto dos prontuários e falta de informações. Nota-se então a necessidade da atenção especial para as gestantes e puérperas, de todas as faixas etárias e realidades socioeconômicas, a fim de propiciar um acompanhamento mais integral e eficaz. Além de todos os aspectos relacionados ao contexto materno, é fundamental analisar o perfil dos parceiros e o impacto dessa correlação. Por fim, o bem estar da mulher deve ser promovido em todos os âmbitos sociais, enfatizando a beleza e feminilidade de uma gestação bem assistida.

\section{REFERÊNCIAS}

1. AGUIAR FAR, et al. Experiência da gravidez entre adolescentes gestantes. Rev. enferm UFPE online. [Internet] 2018; 12(7): 1986-96.

2. ARRAIS AR, ARAÚJO TCCF. Depressão pós-parto: uma revisão sobre fatores de risco e de proteção. Psic., Saúde \& Doenças.Lisboa, 2017; $18: 3$.

3. BONIM SSS, et al. A importância da participação do pai no acompanhamento do pré-natal. Rev. Saberes, Rolim de Moura, 2020; 13: 1.

4. BRASIL. Lei no 8069 , de 13 de julho de 1990. Dispõe sobre a proteção integral à criança e ao adolescente.

5. BRASIL. Ministério da Saúde. Portaria $n^{\circ} 570$ de $1^{\circ}$ de junho de 2000 . Programa de Humanização no Pré-natal e Nascimento. Diário Oficial da União.

6. CARDOSO RF, et al. Educação em saúde na assistência pré-natal: revisão de literatura. Revista Eletrônica Acervo Saúde, 2019; 23(e397-e397).

7. CARDOSO VEPS, et al. A participação do parceiro na rotina pré-natal sob a perspectiva da mulher gestante. Revista de Pesquisa: Cuidado é Fundamental, 2018; 10(3): 856-862.

8. CARNIEL EF, et al. Características das mães adolescentes e de seus recém-nascidos e fatores de risco para a gravidez na adolescência em Campinas, SP, Brasil. Revista Brasileira de Saúde Materno Infantil, 2006; 6(4): 419426.

9. COSTA SP, et al. Transtornos mentais na gravidez e condições do recém-nascido: estudo longitudinal com gestantes assistidas na atenção básica. Ciênc. saúde coletiva. Rio de Janeiro, 2018; 23: 3.

10. DIAS ACG, et al. Gravidez na adolescência: um olhar sobre um fenômeno complexo. Paidéia (Ribeirão Preto), 2010; 20(45): 123-131.

11. FARIAS RV, et al. Gravidez na adolescência e o desfecho da prematuridade: uma revisão integrativa de literatura. Revista Eletrônica Acervo Saúde, 2020; 56: e3977.

12. FISHER RA. On the Interpretation of 2 from Contingency Tables, and the Calculation of $P$, Journal of the Royal Statistical Society, 1922; 85(1): 87-94.

13. GRAVENA AAF, et al. Idade materna e fatores associados a resultados perinatais. Acta Paulista de enfermagem, 2013; 26(2): 130-135.

14. IBGE. Instituto Brasileiro De Geografia e Estatística. Censo demográfico 2000: nupcialidade e fecundidade: resultados da amostra. Rio de Janeiro; 2003.

15. IFRS. INSTITUTO FEDERAL RIO GRANDE DO SUL -. Boletim SAtS: Pais e Filhos. Seção de Atenção à Saúde do Servidor. Ed. 2018; 5. 
16. KASSAR SB, et al. Comparações das condições socioeconômicas e reprodutivas entre mães adolescentes e adultas jovens em três maternidades públicas de Maceió, Brasil. Revista Brasileira de Saúde Materno Infantil, 2006; 6(4): 397-403.

17. MARCHETTI JR, et al. A importância do pré-natal. Anuário Pesquisa e Extensão Unoesc Xanxerê, $2020 ; 5$ : e24175.

18. PEARSON K. The fundamental problem of practical statistics. Biometrika, 1920; 13(1): 1-16.

19. ROSA CQ, et al. Factors associated with lack of prenatal care in a large municipality. Rev Saúde Pública, 2014; 48(6): 977-984.

20. SALDANHA BL. Dificuldades enfrentadas por gestantes adolescentes em aderir ao pré-natal. Revista Eletrônica Acervo Saúde, 2020; 12(9): e4160.

21. SERRUYA SJ, et al. Avaliação preliminar do Programa de Humanização no Pré natal e Nascimento no Brasil. Rev Bras Ginecol Obstet., 2004; 26(7): 517-25.

22. SPINDOLA T, et al. Perfil epidemiológico de mulheres atendidas na consulta do pré-natal de um hospital universitário. Revista da Escola de Enfermagem da USP. 2006; 40(3): 381-88.

23. VIELLAS EF, et al. Assistência pré-natal no Brasil. Cad. Saúde Pública, Rio de Janeiro, 2014; 30(supl. 1): S85S100. 